\title{
Stability Analysis for a Fractional HIV Infection Model with Nonlinear Incidence
}

\author{
Linli Zhang, ${ }^{1}$ Gang Huang, ${ }^{2}$ Anping Liu, ${ }^{2}$ and Ruili Fan ${ }^{3}$ \\ ${ }^{1}$ Department of Basic Course, Haikou College of Economics, Haikou 571127, China \\ ${ }^{2}$ School of Mathematics and Physics, China University of Geosciences, Wuhan 430074, China \\ ${ }^{3}$ Department of Mathematics and Statistics, University of Helsinki, 00014 Helsinki, Finland \\ Correspondence should be addressed to Anping Liu; wh_apliu@sina.com
}

Received 30 September 2014; Accepted 16 December 2014

Academic Editor: Bai-Lian Larry Li

Copyright (C) 2015 Linli Zhang et al. This is an open access article distributed under the Creative Commons Attribution License, which permits unrestricted use, distribution, and reproduction in any medium, provided the original work is properly cited.

We introduce the fractional-order derivatives into an HIV infection model with nonlinear incidence and show that the established model in this paper possesses nonnegative solution, as desired in any population dynamics. We also deal with the stability of the infection-free equilibrium, the immune-absence equilibrium, and the immune-presence equilibrium. Numerical simulations are carried out to illustrate the results.

\section{Introduction}

1.1. The History of Fractional Calculus. Fractional calculus which is a branch of mathematical analysis extends derivatives and integrals to an arbitrary order (real, even, or complex order). The study on fractional calculus started at the end of seventeenth century and the first reference was proposed by Leibniz and LHospital in 1695, in which halforder derivative was mentioned. The emergence of fractional calculus aroused interest of some famous mathematicians such as Lacroix, Lagrange, Fourier, and Laplace. J. L. Lagrange developed the law of exponents for differential operators in 1772, which promoted indirectly the development of fractional calculus. P. S. Laplace defined a fractional derivative by means of integral in 1812 and S. F. Lacroix and J. B. J. Fourier in succession mentioned the arbitrary order derivative, respectively, in 1819 and in 1822. However, the basic theory of fractional calculus was established with the studies of Liouville, Grunwald, Letnikov, and Riemann until the end of the nineteenth century.

At the initial stage, the development of fractional calculus had been restricted to the pure mathematics research. Until the nineteen nineties, the theory of fractional calculus was applied to nature and social sciences. In the field of anomalous diffusion, the researchers applied fractional calculus to describe the diffusion processes which do not conform to the brown motion and proposed the fractional anomalous diffusion model. According to the theoretical analysis and the experimental data or results, they found that the fractional model is more reasonable to describe these processes [1]. Afterwards, many mathematicians and applied researchers also have tried to demonstrate applications of fractional differentials in the areas of non-Newtonian fluids [2], signal processing [3-5], viscoelasticity [6, 7], fluid-dynamic traffic model [8], colored noise [9], bioengineering [10], solid mechanics [11], continuum and statistical mechanics [12], and economics [13] and brought new research view for those fields.

1.2. Mathematical Modeling. In biology, it has been deduced that the membranes of cells of biological organism have fractional-order electrical conductance [14]; hence some mathematical models which describe cells behavior are classified into groups of non-integer-order models. In the field of rheology, fractional-order derivatives embody essential features of cell rheological behavior and have enjoyed greatest success [15]. Some researchers also found that fractional ordinary differential equations are naturally related to systems with memory which exists in most biological systems. And fractional-order equations are also closely related to fractals, which are abundant in biological systems. 
Mathematical models have been proven valuable in understanding the dynamics of viral infection. Although a large number of works on modeling the dynamics of viral infection have been done [16-20], it has been restricted to integer-order (delay) differential equations. In recent years, it has turned out that many phenomena in virus infection can be described very successfully by the models using fractional-order differential equations [21, 22]. Motivated by these references, in this paper, we will consider a fractionalorder HIV model.

Among a large number of virus infection models described with integer-order or delay differential equations, a typical model is given as the following simple threedimensional system:

$$
\begin{gathered}
x^{\prime}(t)=\lambda-d x(t)-\beta x(t) v(t), \\
y^{\prime}(t)=\beta x(t) v(t)-p y(t), \\
v^{\prime}(t)=k y(t)-u v(t) .
\end{gathered}
$$

Here $x(t)$ represents the concentration of uninfected cells at time $t ; y(t)$ represents the concentration of infected cells that produce virus at time $t ; v(t)$ represents the concentration of viruses at time t. $d(d>0)$ and $\beta(\beta>0)$ are the death rate of uninfected cells and the rate constant characterizing infection of the cells, respectively. $p(p>0)$ is the death rate of infected cells due to either the virus or the immune system. Free virus is produced from infected cells at the rate $k y(t)$ and removed at rate $u v(t)$.

The infection rate in model (1) is usually assumed to be bilinear with respect to virus $v(\mathrm{t})$ and uninfected target cells $x(t)$. However, there are some evidences which show that a bilinear infection might not be an effective assumption when the number of target cells is large enough [23-25]. And Huang et al. [17] incorporated Holling type II functional response infection rate $g(x(t), v(t))$ into basic virus dynamics (1), which was expressed as

$$
g(x(t), v(t))=\frac{\beta x(t) v(t)}{1+q x(t)},
$$

where $q(q \geq 0)$ is a constant. Hence, system (1) can be modified into the following system:

$$
\begin{gathered}
x^{\prime}(t)=\lambda-d x(t)-\frac{\beta x(t) v(t)}{1+q x(t)}, \\
y^{\prime}(t)=\frac{\beta x(t) v(t)}{1+q x(t)}-p y(t), \\
v^{\prime}(t)=k y(t)-u v(t) .
\end{gathered}
$$

The immune response following viral infection is universal and necessary to eliminate or control the disease. Antibodies, cytokines, natural killer cells, B cells, and T cells are all essential components of a normal immune response to viral infection. Since cytotoxic T lymphocytes (CTLs) play a critical role in antiviral defense by attacking the virusinfected cells, Huang et al. [17] considered the immune response to model (3) with $z^{\prime}(t)=c y(t) z(t)-b z(t)$.
System (3) with $z^{\prime}(t)=c y(t) z(t)-b z(t)$ can be further simplified if we take into consideration the fact that an average life span of viral particles is usually significantly shorter than one of the infected cells. It can be assumed, therefore, that, compared with a "slow" variation of the infected cells level, the virus load $v(t)$ relatively quickly reaches a quasiequilibrium level. The equality $\dot{v}(t)=0$ holds in the quasiequilibrium state and hence $v(t)=k y(t) / u$. This assumption is referred to as "separation of time scales" and is in common use in the virus dynamics [18]. We have to stress that this assumption does not imply that the virus concentration $v(t)$ remains constant; on the contrary, it is assumed to be proportional to the varying concentration of infected cells $y(t)$. Accordingly, system (3) with $z^{\prime}(t)=$ $c y(t) z(t)-b z(t)$ can now be reformulated as a system of three differential equations:

$$
\begin{gathered}
x^{\prime}(t)=\lambda-d x(t)-\frac{\beta x(t) y(t)}{1+q x(t)}, \\
y^{\prime}(t)=\frac{\beta x(t) y(t)}{1+q x(t)}-a y(t)-p y(t) z(t), \\
z^{\prime}(t)=c y(t) z(t)-b z(t) .
\end{gathered}
$$

Further we introduce the fractional order into model (4) and a new system can be described by the following set of FODEs of order $\alpha>0$ :

$$
\begin{gathered}
x^{\alpha}(t)=\lambda-d x-\frac{\beta x y}{1+q x}, \\
y^{\alpha}(t)=\frac{\beta x y}{1+q x}-a y-p y z, \\
z^{\alpha}(t)=c y z-b z, \\
x(0)=x_{0}, \quad y(0)=y_{0}, \quad z(0)=z_{0},
\end{gathered}
$$

where the immune response is assumed to get stronger at a rate $c y z$, which is proportional to the number of infected cells and their current concentration; the immune response decays exponentially at a rate $b z$, which is proportional to their current concentration; the parameter $q$ expresses the efficacy of nonlytic component.

The paper is organized as follows. In the next section, we give two definitions and two lemmas about fractional calculus. In Section 3, we show the nonnegativeness of the solutions of system (5) with initial condition (6). In Section 4, we give a detailed stability analysis for three equilibria. Finally numerical simulations are presented to illustrate the obtained results in Section 5.

\section{Fractional Calculus}

Definition 1 (see [26]). The Riemann-Liouville (R-L) fractional integral operator of order $\alpha>0$ of a function $f: R^{+} \rightarrow$ $R$ is defined as

$$
I^{\alpha} f(x)=\frac{1}{\Gamma(\alpha)} \int_{0}^{x}(x-t)^{\alpha-1} f(t) d t .
$$


Here $\Gamma(\cdot)$ is the Euler gamma function which is defined as

$$
\Gamma(n)=\int_{0}^{\infty} t^{n-1} e^{-t} d t
$$

This function is generalization of a factorial in the following form:

$$
\Gamma(n)=(n-1) !
$$

Definition 2 (see [26]). The Caputo (C) fractional derivative of order $\alpha>0, n-1<\alpha<n, n \in N$, is defined as

$$
D^{\alpha} f(t)=I^{n-\alpha} D^{n} f(t)=\frac{1}{\Gamma(n-\alpha)} \int_{0}^{t} \frac{f^{(n)}(s)}{(t-s)^{\alpha+1-n}} d s,
$$

where the function $f(t)$ has absolutely continuous derivatives up to order $(n-1)$. In particular, when $0<\alpha<1$, one has

$$
D^{\alpha} f(t)=\frac{1}{\Gamma(1-\alpha)} \int_{0}^{t} \frac{f^{\prime}(s)}{(t-s)^{\alpha}} d s .
$$

In this paper we use Caputo fractional derivative definition. The main advantage of Caputo's definition is that the initial conditions for fractional differential equations with Caputo derivatives take the same form as that for integerorder differential equations.

Lemma 3 (see [26]). Consider the following commensurate fractional-order system:

$$
\begin{gathered}
D^{\alpha} x=f(x), \\
x(0)=x_{0},
\end{gathered}
$$

with $0<\alpha \leq 1$ and $x \in R^{n}$. The equilibrium points of system (12) are calculated by solving the following equation: $f(x)=0$. These points are locally asymptotically stable if all eigenvalues $r_{i}$ of Jacobian matrix $J=\partial f / \partial x$ evaluated at the equilibrium points satisfy

$$
\left|\arg \left(r_{i}\right)\right|>\frac{\theta \pi}{2}
$$

Definition 4 (see [27]). The discriminant $D(f)$ of a polynomial

$$
f(x)=x^{n}+c_{1} x^{n-1}+c_{2} x^{n-2}+\cdots+c_{n}
$$

is defined by $D(f)=(-1)^{n(n-1) / 2} R\left(f, f^{\prime}\right)$, where $f^{\prime}$ is the derivative of $f$. If $g(x)=x^{l}+d_{1} x^{l-1}+d_{2} x^{l-2}+\cdots+d_{n}, R(f, g)$ is the determinant of the corresponding Sylvester $(n+l) \otimes(n+l)$ matrix. The Sylvester matrix is formed by filling the matrix beginning with the upper left corner with the coefficients of $f(x)$ and then shifting down one row and one column to the right and filling in the coefficients starting there until they hit the right side. The process is then repeated for the coefficients of $g(x)$.

Lemma 5 (see [27]). For the polynomial equation,

$$
P(\lambda)=\lambda^{n}+h_{1} \lambda^{n-1}+h_{2} \lambda^{n-2}+\cdots+h_{n}=0,
$$

the conditions which make all the roots of (15) satisfy (13) are displayed as follows: (i) for $n=1$, the condition is $h_{1}>0$;

(ii) for $n=2$, the conditions are either Routh-Hurwitz conditions or

$$
\begin{gathered}
h_{1}<0, \quad 4 h_{2}>\left(h_{1}\right)^{2}, \\
\left|\tan ^{-1}\left(\frac{\sqrt{4 h_{2}-\left(h_{1}\right)^{2}}}{h_{1}}\right)\right|>\frac{\alpha \pi}{2}
\end{gathered}
$$

(iii) for $n=3$,

(a) if the discriminant of $P(\lambda), D(P)$ is positive, then Routh-Hurwitz conditions are the necessary and sufficient conditions; that is, $h_{1}>0, h_{3}>0$, and $h_{1} h_{2}>h_{3}$ if $D(P)>0$

(b) if $D(P)<0, h_{1} \geq 0, h_{2} \geq 0$, and $h_{3}>0$, then (13) for (15) holds when $\alpha<2 / 3$;

(c) if $D(P)<0, h_{1}<0$, and $h_{2}<0$, then (13) for (15) holds when $\alpha>2 / 3$;

(d) if $D(P)<0, h_{1}>0, h_{2}>0$, and $h_{1} h_{2}=h_{3}$, then (13) for (15) holds for all $\alpha \in[0,1)$.

\section{Nonnegative Solutions}

Let $R_{+}^{3}=\left\{W \in R^{3}: W \geq 0\right\}$ and $W(t)=(x(t), y(t), z(t))^{T}$. For the proof of the theorem about nonnegative solutions, we would need the following lemma.

Lemma 6 (see [28] generalized mean value theorem). Let $f(x) \in C[a, b]$ and $D^{\alpha} f(x) \in C[a, b]$ for $0<\alpha \leq 1$. Then one has

$$
f(x)=f(a)+\frac{1}{\Gamma(\alpha)} D^{\alpha} f(\xi)(x-a)^{\alpha},
$$

with $0 \leq \xi \leq x, \forall x \in(a, b]$, where $\Gamma(x)=\int_{0}^{+\infty} t^{x-1} e^{-t} d t$.

Remark 7. Suppose that $f(x) \in C[a, b]$ and $D^{\alpha} f(x) \in$ $C[a, b]$, for $0<\alpha \leq 1$. It is clear from Lemma 6 that if $D^{\alpha} f(x) \geq 0, \forall x \in(a, b)$, then $f(x)$ is nondecreasing for each $x \in[a, b]$. If $D^{\alpha} f(x) \leq 0, \forall x \in(a, b)$, then $f(x)$ is nonincreasing for each $x \in[a, b]$.

We now prove the main theorem.

Theorem 8. There is a unique solution for the initial value problem (5) with (6) and the solution remains in $R_{+}^{3}$.

Proof. From Theorem 3.1 and Remark 3.2 of [29], we obtain the solution on $(0, \infty)$ solving the initial value problem $(5)$ with (6) which is not only existent but also unique. Next, we will show the nonnegative orthant $R_{+}^{3}$ is a positively invariant region. What is needed for this is to show that, on each hyperplane bounding the nonnegative orthant, the vector field points into $R_{+}^{3}$. From (5), we find

$$
\left.x^{\alpha}(t)\right|_{x=0}=\lambda \geq 0,\left.\quad y^{\alpha}(t)\right|_{y=0}=0,\left.\quad z^{\alpha}(t)\right|_{z=0}=0 .
$$

By Remark 7, the solution will remain in $R_{+}^{3}$. 


\section{Equilibrium States and Their Stability}

In this section, we investigate the stability of the fractionalorder model of HIV infection of CD4 + T cells, that is, system (5) with (6). Consider the initial value problem (5) with (6) with $\alpha$ satisfying $0<\alpha \leq 1$.

In order to obtain the equilibria of system (5), we set $x^{\alpha}(t)=0, y^{\alpha}(t)=0$, and $z^{\alpha}(t)=0$ and we have

$$
\begin{gathered}
\lambda-d x-\frac{\beta x y}{1+q x}=0, \\
\frac{\beta x y}{1+q x}-a y-p y z=0, \\
c y z-b z=0 .
\end{gathered}
$$

System (5) has three types of relevant nonnegative equilibrium states. System (5) always has an infection-free equilibrium $E_{0}=\left(x_{0}, 0,0\right)$, where

$$
x_{0}=\frac{\lambda}{d}
$$

which means that the infected cells are cleared. The basic reproductive number of the viruses for system (5) is given by

$$
R_{0}=\frac{\lambda \beta}{a(d+q \lambda)} .
$$

This number describes the average number of newly generated infected cells from one infected cell at the beginning of the infection process. When $R_{0}>1$, in addition to the infection-free equilibrium $E_{0}$, there is an immune-absence equilibrium $E_{1}=\left(x_{1}, y_{1}, z_{1}\right)$, where

$$
x_{1}=\frac{a}{\beta-a q}, \quad y_{1}=\frac{d+q \lambda}{\beta-a q}\left(R_{0}-1\right), \quad z_{1}=0 .
$$

Note that $R_{0}>1$ implies $\beta>$ aq. When the HIV infection is in the immune-absence equilibrium $E_{1}$, the infected cells and virus exist but the immune response is not activated yet. Further, we denote

$$
R_{1}=R_{0}-\frac{b(\beta-a q)}{c(d+q \lambda)}
$$

Note that $R_{1}=((\beta-a q) / c(d+q \lambda))\left(c y_{1}-b\right)+1$, which implies that $R_{1}>1$ is equivalent to $c y_{1} / b>1$. The latter $c y_{1} / b$ is seen as the immune reproductive number, which expresses the average number of activated CTLs generated from one CTL during its life time $1 / b$ through the stimulation of the infected cells $y_{1}$. It is reasonable that immune response is activated in the case where $R_{1}>1$. When $R_{1}>1$, in addition to the infection-free equilibrium $E_{0}$ and the immune-absence equilibrium $E_{1}$, there is an interior immune-presence equilibrium $E^{*}=\left(x^{*}, y^{*}, z^{*}\right)$, where

$$
\begin{gathered}
x^{*}=\frac{-(b \beta+c d-c q \lambda)+\sqrt{(b \beta+c d-c q \lambda)^{2}+4 c^{2} d q \lambda}}{2 c d q}, \\
y^{*}=\frac{b}{c}, \quad z^{*}=\frac{c}{b p}\left(\lambda-d x^{*}\right)-\frac{a}{p} .
\end{gathered}
$$

$E^{*}$ expresses the state where CTLs immune response is present.

Next, we establish the local asymptotic stability of model (5) by the characteristic equation.

Theorem 9. Consider system (5).

(1) The infection-free equilibrium $E_{0}$ is locally asymptotically stable if $R_{0}<1$.

(2) If $R_{0}>1$, the equilibrium $E_{0}$ is unstable, and if $R_{0}=1$, it is a critical case.

Proof. The characteristic equation for the infection-free equilibrium $E_{0}$ is given as follows:

$$
\operatorname{det}\left(\begin{array}{ccc}
-d-r & -\frac{\beta x_{0}}{1+q x_{0}} & 0 \\
0 & \frac{\beta x_{0}}{1+q x_{0}}-a-r & 0 \\
0 & 0 & -b-r
\end{array}\right)=0 .
$$

It is reduced to

$$
(r+d)(r+b)\left(r+a-\frac{\beta x_{0}}{1+q x_{0}}\right)=0 .
$$

It is clear that (26) has the characteristic roots $r_{1}=-d<0$ which means $\left|\arg r_{1}\right|=\pi>\alpha(\pi / 2), r_{2}=-b<0$ which means $\left|\arg r_{2}\right|=\pi>\alpha(\pi / 2)$, and $r_{3}=\left(\beta x_{0} /\left(1+q x_{0}\right)\right)-a=$ $a\left(R_{0}-1\right)$. Since the imaginary part of characteristic root $r_{3}$ is zero, $R_{0}<1$ which means $\left|\arg r_{3}\right|=\pi>\alpha(\pi / 2)$ is necessary and sufficient to ensure the local asymptotic stability of $E_{0}$. If $R_{0}>1,\left|\arg r_{3}\right|=0<\alpha(\pi / 2)$; hence $E_{0}$ is unstable. If $R_{0}=1$, $r_{3}=0$, which is a critical case.

\section{Theorem 10. Consider system (5).}

(1) The immune-free equilibrium $E_{1}$ is locally asymptotically stable if $R_{1}<1$.

(2) If $R_{1}>1$, the immune-free equilibrium $E_{1}$ is unstable; if $R_{1}=1$, it is a critical state.

Proof. The characteristic equation for the immune-free equilibrium $E_{1}$ is given as follows:

$$
\operatorname{det}\left(\begin{array}{ccc}
-d-\frac{\beta y_{1}}{\left(1+q x_{1}\right)^{2}}-r & -\frac{\beta x_{1}}{1+q x_{1}} & 0 \\
\frac{\beta y_{1}}{\left(1+q x_{1}\right)^{2}} & \frac{\beta x_{1}}{1+q x_{1}}-a-r & -p y_{1} \\
0 & 0 & c y_{1}-b-r
\end{array}\right)
$$$$
=0 \text {. }
$$

Using $\beta x_{1} /\left(1+q x_{1}\right)=a$, it is reduced to

$$
\left(r+b-c y_{1}\right)\left[r^{2}+\left(d+\frac{\beta y_{1}}{\left(1+q x_{1}\right)^{2}}\right) r+\frac{a \beta y_{1}}{\left(1+q x_{1}\right)^{2}}\right]=0 .
$$


The root of (28) $r_{1}=c y_{1}-b$ is negative and $\left|\arg r_{1}\right|=\pi>$ $\alpha(\pi / 2)$ when $R_{1}<1$, positive and $\left|\arg r_{1}\right|=0<\alpha(\pi / 2)$ when $R_{1}>1$, and zero when $R_{1}=1$, which is a critical case.

Now, we consider the equation

$$
r^{2}+\left(d+\frac{\beta y_{1}}{\left(1+q x_{1}\right)^{2}}\right) r+\frac{a \beta y_{1}}{\left(1+q x_{1}\right)^{2}}=0 .
$$

Denote

$$
\begin{aligned}
& B=\frac{\beta y_{1}}{\left(1+q x_{1}\right)^{2}}, \\
& C=\frac{a \beta y_{1}}{\left(1+q x_{1}\right)^{2}} .
\end{aligned}
$$

Since $B>0$ and $C>0$, (29) has two negative real roots and we denote them by $r_{2}$ and $r_{3}$. It is easy to see $\left|\arg r_{2}\right|=\pi>$ $\alpha(\pi / 2)$ and $\left|\arg r_{3}\right|=\pi>\alpha(\pi / 2)$. Hence, when $R_{1}<1$, the immune-free equilibrium $E_{1}$ is locally asymptotically stable, when $R_{1}>1, E_{1}$ is unstable, and when $R_{1}=1$, it is a critical case.

To discuss the local stability of the immune-present equilibrium state $E^{*}$, we consider the linearized system of (5) at $E^{*}$. The Jacobian matrix at $E^{*}$ is given by

$$
J\left(E^{*}\right)=\left(\begin{array}{ccc}
-d-\frac{\beta y^{*}}{\left(1+q x^{*}\right)^{2}} & -\frac{\beta x^{*}}{1+q x^{*}} & 0 \\
\frac{\beta y^{*}}{\left(1+q x^{*}\right)^{2}} & 0 & -p y^{*} \\
0 & c z^{*} & 0
\end{array}\right) .
$$

The characteristic equation of the linearized system is

$$
P(r)=r^{3}+a_{1} r^{2}+a_{2} r+a_{3}=0,
$$

where

$$
\begin{gathered}
a_{1}=d+\frac{\beta y^{*}}{\left(1+q x^{*}\right)^{2}}>0, \\
a_{2}=c p y^{*} z^{*}+\frac{\beta^{2} x^{*} y^{*}}{\left(1+q x^{*}\right)^{3}}>0, \\
a_{3}=c p y^{*} z^{*}\left(d+\frac{\beta y^{*}}{\left(1+q x^{*}\right)^{2}}\right)>0, \\
a_{1} a_{2}-a_{3}=\frac{\beta^{2} x^{*} y^{*}}{\left(1+q x^{*}\right)^{3}}\left(d+\frac{\beta y^{*}}{\left(1+q x^{*}\right)^{2}}\right)>0 .
\end{gathered}
$$

Based on Definition 4, we obtain the discriminant of (32)

$$
\begin{aligned}
D(P) & =-\left|\begin{array}{ccccc}
1 & a_{1} & a_{2} & a_{3} & 0 \\
0 & 1 & a_{1} & a_{2} & a_{3} \\
3 & 2 a_{1} & a_{2} & 0 & 0 \\
0 & 3 & 2 a_{1} & a_{2} & 0 \\
0 & 0 & 3 & 2 a_{1} & a_{2}
\end{array}\right| \\
& =18 a_{1} a_{2} a_{3}+\left(a_{1} a_{2}\right)^{2}-4 a_{1}^{3} a_{3}-4 a_{2}^{3}-27 a_{3}^{2} .
\end{aligned}
$$

Using the result (iii) of Lemmas 5 and 3, we have the following theorem.

Theorem 11. Consider system (5). Under the condition of $R_{1}>$ 1 ,

(1) if the discriminant of $P(r), D(P)$ is positive, namely, $D(P)>0$, then the immune-present equilibrium $E^{*}$ is locally asymptotically stable for $0<\alpha \leq 1$;

(2) if $D(P)<0$, then the immune-present equilibrium $E^{*}$ is locally asymptotically stable for $0<\alpha<2 / 3$.

\section{Numerical Method}

Atanackovic and Stankovic introduced a numerical method to solve the single linear FDE in 2004 [1]. A few years later, they developed again a method to solve the nonlinear FDE [30]. It was shown that the fractional derivative of a function $f(t)$ with order $\alpha$ satisfying $0<\alpha<1$ may be expressed as

$$
\begin{aligned}
D^{\alpha} f(t) & \\
= & \frac{1}{\Gamma(2-\alpha)} \\
& \times\left\{\frac{f^{(1)}(t)}{t^{\alpha-1}}\left[1+\sum_{m=1}^{\infty} \frac{\Gamma(m-1+\alpha)}{\Gamma(\alpha-1) m !}\right]\right. \\
& \quad\left[\frac{\alpha-1}{t^{\alpha}} f(t)+\sum_{m=2}^{\infty} \frac{\Gamma(m-1+\alpha)}{\Gamma(\alpha-1)(m-1) !}\right. \\
& \left.\left.\times\left(\frac{f(t)}{t^{\alpha}}+\frac{V_{m}(f)(t)}{t^{m-1+\alpha}}\right)\right]\right\},
\end{aligned}
$$

where

$$
V_{m}(f)(t)=-(m-1) \int_{0}^{t} \tau^{m-2} f(\tau) d \tau, \quad m=2,3, \ldots,
$$

with the following properties:

$$
\frac{d}{d t} V_{m}(f)=-(m-1) t^{m-2} f(t), \quad m=2,3, \ldots
$$

We approximate $D^{\alpha} f(t)$ by using $M$ terms in sums appearing in (35) as follows:

$$
\begin{aligned}
D^{\alpha} f(t) & \\
\simeq & \frac{1}{\Gamma(2-\alpha)} \\
& \times\left\{\frac{f^{(1)}(t)}{t^{\alpha-1}}\left[1+\sum_{m=1}^{M} \frac{\Gamma(m-1+\alpha)}{\Gamma(\alpha-1) m !}\right]\right. \\
& \quad\left[\frac{\alpha-1}{t^{\alpha}} f(t)+\sum_{m=2}^{M} \frac{\Gamma(m-1+\alpha)}{\Gamma(\alpha-1)(m-1) !}\right. \\
& \left.\left.\times\left(\frac{f(t)}{t^{\alpha}}+\frac{V_{m}(f)(t)}{t^{m-1+\alpha}}\right)\right]\right\} .
\end{aligned}
$$


We can rewrite (38) as follows:

$$
\begin{aligned}
D^{\alpha} f(t) \simeq & \Omega(\theta, t, M) f^{(1)}(t)+\Phi(\theta, t, M) f(t) \\
& +\sum_{m=2}^{M} A(\theta, t, m) \frac{V_{m}(f)(t)}{t^{m-1+\alpha}},
\end{aligned}
$$

where

$$
\begin{gathered}
\Omega(\alpha, t, M)=\frac{1+\sum_{m=1}^{M}(\Gamma(m-1+\alpha) / \Gamma(\alpha-1) m !)}{\Gamma(2-\alpha) t^{\alpha-1}}, \\
R(a, t)=\frac{1-\alpha}{t^{\alpha} \Gamma(2-\alpha)} \\
A(\alpha, t, m)=-\frac{\Gamma(m-1+\alpha)}{\Gamma(2-\alpha) \Gamma(\alpha-1)(m-1) !} \\
\Phi(\alpha, t, M)=R(a, t)+\sum_{m=2}^{M} \frac{A(\alpha, t, m)}{t^{\alpha}}
\end{gathered}
$$

We set

$$
\begin{aligned}
\Theta_{1}(t) & =x(t), & & \Theta_{m}(t)=V_{m}(x)(t), \\
\Theta_{M+1}(t) & =y(t), & & \Theta_{M+m}(t)=V_{m}(y)(t), \\
\Theta_{2 M+1}(t) & =z(t), & & \Theta_{2 M+m}(t)=V_{m}(z)(t),
\end{aligned}
$$

for $m=2,3, \ldots$. We can rewrite system (19) as the following form:

$$
\begin{aligned}
& \Omega(\alpha, t, M) \Theta_{1}^{\prime}(t)+\Phi(\alpha, t, M) \Theta_{1}(t)+\sum_{m=2}^{M} A(\alpha, t, m) \frac{\Theta_{m}(t)}{t^{m-1+\alpha}} \\
& =\lambda-d \Theta_{1}(t)-\frac{\beta \Theta_{1}(t) \Theta_{M+1}(t)}{1+q \Theta_{1}(t)}
\end{aligned}
$$$$
\Omega(\alpha, t, M) \Theta_{M+1}^{\prime}(t)+\Phi(\alpha, t, M) \Theta_{M+1}(t)
$$$$
+\sum_{m=2}^{M} A(\alpha, t, m) \frac{\Theta_{M+m}(t)}{t^{m-1+\alpha}}
$$$$
=\frac{\beta \Theta_{1}(t) \Theta_{M+1}(t)}{1+q \Theta_{1}(t)}-a \Theta_{M+1}(t)-p \Theta_{M+1}(t) \Theta_{2 M+1}(t),
$$

$\Omega(\alpha, t, M) \Theta_{2 M+1}^{\prime}(t)+\Phi(\alpha, t, M) \Theta_{2 M+1}(t)$

$$
\begin{aligned}
& +\sum_{m=2}^{M} A(\alpha, t, m) \frac{\Theta_{2 M+m}(t)}{t^{m-1+\alpha}} \\
= & c \Theta_{M+1}(t) \Theta_{2 M+1}(t)-b \Theta_{2 M+1}(t),
\end{aligned}
$$

where

$$
\begin{array}{r}
\Theta_{m}(t)=-(m-1) \int_{0}^{t} \tau^{m-2} \Theta_{1}(\tau) d \tau, \\
\Theta_{M+m}(t)=-(m-1) \int_{0}^{t} \tau^{m-2} \Theta_{M+1}(\tau) d \tau, \\
\Theta_{2 M+m}(t)=-(m-1) \int_{0}^{t} \tau^{m-2} \Theta_{2 M+1}(\tau) d \tau,
\end{array}
$$

$$
m=2,3, \ldots, M \text {. }
$$

Now we can rewrite (39) and (42) as the following form:

$$
\begin{aligned}
& \Theta_{1}^{\prime}(t)=\frac{1}{\Omega(\alpha, t, M)}\left(\lambda-(d+\Phi(\alpha, t, M)) \Theta_{1}(t)\right. \\
& -\frac{\beta \Theta_{1}(t) \Theta_{M+1}(t)}{1+q \Theta_{1}(t)} \\
& \left.-\sum_{m=2}^{M} A(\alpha, t, m) \frac{\Theta_{m}(t)}{t^{m-1+\alpha}}\right), \\
& \Theta_{m}^{\prime}(t)=-(m-1) t^{m-2} \Theta_{1}(t), \quad m=2,3, \ldots, M, \\
& \Theta_{M+1}^{\prime}(t)=\frac{1}{\Omega(\alpha, t, M)}\left(\frac{\beta \Theta_{1}(t) \Theta_{M+1}(t)}{1+q \Theta_{1}(t)}\right. \\
& -(a+\Phi(\alpha, t, M)) \Theta_{M+1}(t) \\
& \text { - } p \Theta_{M+1}(t) \Theta_{2 M+1}(t) \\
& \left.-\sum_{m=2}^{M} A(\alpha, t, m) \frac{\Theta_{M+m}(t)}{t^{m-1+\alpha}}\right),
\end{aligned}
$$

$$
\begin{array}{r}
\Theta_{M+m}^{\prime}(t)=-(m-1) t^{m-2} \Theta_{M+1}(t), \quad m=2,3, \ldots, M, \\
\Theta_{2 M+1}^{\prime}(t)=\frac{1}{\Omega(\alpha, t, M)}\left(c \Theta_{M+1}(t) \Theta_{2 M+1}(t)\right. \\
-(b+\Phi(\alpha, t, M)) \Theta_{2 M+1}(t) \\
\left.-\sum_{m=2}^{M} A(\alpha, t, m) \frac{\Theta_{2 M+m}(t)}{t^{m-1+\alpha}}\right),
\end{array}
$$

$$
\Theta_{2 M+m}^{\prime}(t)=-(m-1) t^{m-2} \Theta_{2 M+1}(t), \quad m=2,3, \ldots, M,
$$

with the following initial conditions:

$$
\begin{gathered}
\Theta_{1}(\delta)=x_{0}, \quad \Theta_{m}(\delta)=0, \quad m=2,3, \ldots, M, \\
\Theta_{M+1}(\delta)=y_{0}, \quad \Theta_{M+m}(\delta)=0, \quad m=2,3, \ldots, M, \\
\Theta_{2 M+1}(\delta)=z_{0}, \quad \Theta_{2 M+m}(\delta)=0, \quad m=2,3, \ldots, M .
\end{gathered}
$$

Now we consider the numerical solution of system of ordinary differential equations (44) with the initial conditions (45) by using the well-known Runge-Kutta method of fourth order. 


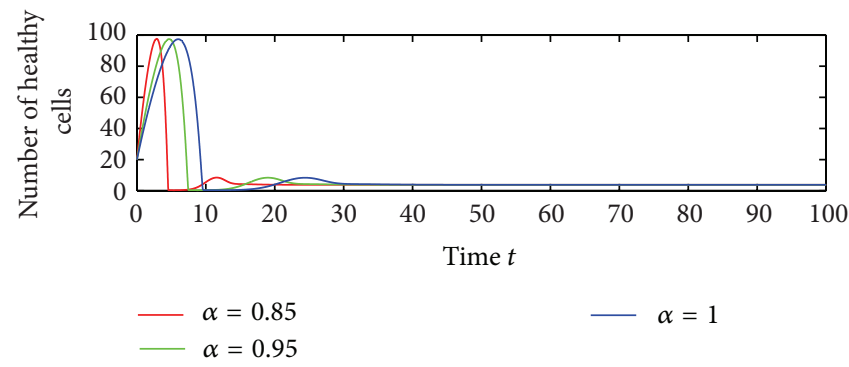

(a) Time evolution of the state variables

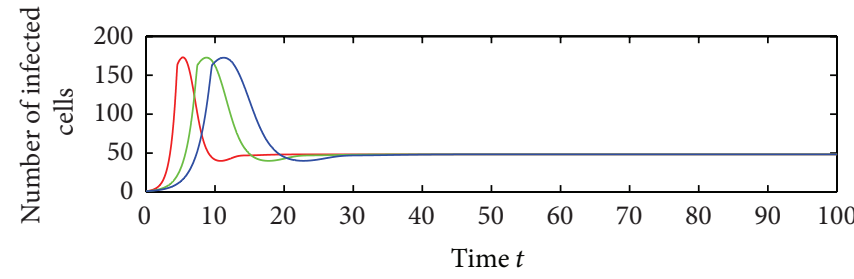

$\alpha=0.85$

$\alpha=0.95$

(b) Time evolution of the state variables

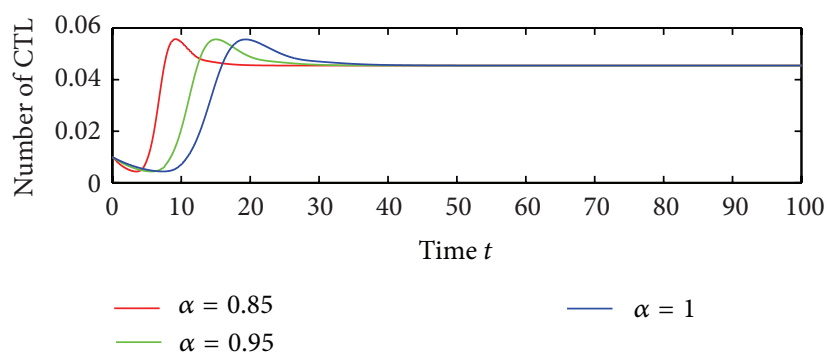

(c) Time evolution of the state variables

Figure 1: The diagrams show the time evolution of the trajectory of system (5) for healthy cells $x(t)$, infected cells $y(t)$, and the CTL $z(t)$, respectively, with respect to $\alpha=0.85, \alpha=0.95$, and $\alpha=1$, in condition of $D(P)>0$. The red lines correspond to $x(t)$ in (a), $y(t)$ in (b), and $z(t)$ in (c) for $\alpha=0.85$; the green lines correspond to $x(t)$ in (a), $y(t)$ in (b), and $z(t)$ in (c) for $\alpha=0.95$ and the blue lines for $\alpha=1$.

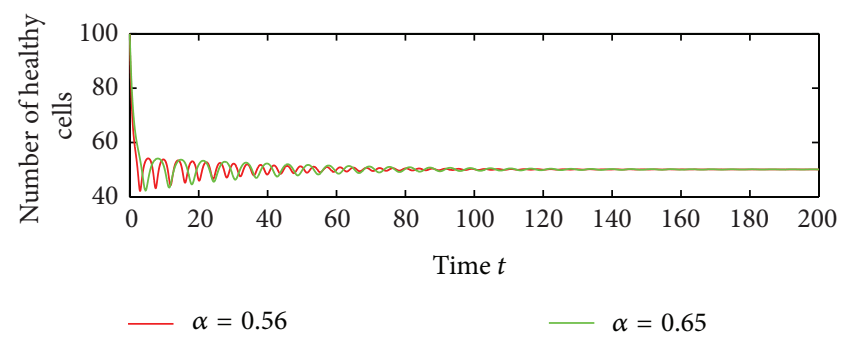

(a) Time evolution of the state variables

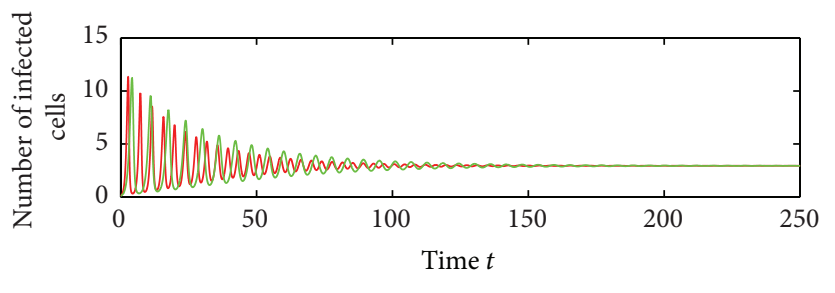

$\alpha=0.56$

(b) Time evolution of the state variables

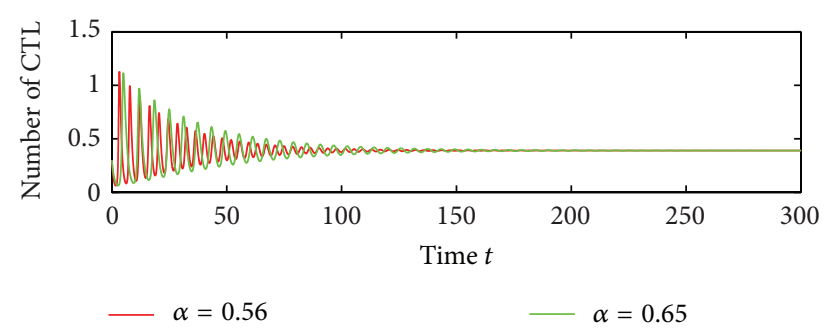

(c) Time evolution of the state variables

FIGURE 2: The diagrams show the time evolution of the trajectory of system (5) for healthy cells $x(t)$, infected cells $y(t)$, and the CTL $z(t)$, respectively, with respect to $\alpha=0.56$ and $\alpha=0.65$, in condition of $D(P)<0$. The red lines correspond to $x(t)$ in $(\mathrm{a}), y(t)$ in (b), and $z(t)$ in (c) for $\alpha=0.56$; the green lines correspond to $x(t)$ in (a), $y(t)$ in (b), and $z(t)$ in (c) for $\alpha=0.65$.

\section{Numerical Simulation and Discussion}

Firstly, by using GEM (generalized Euler method) [21], we simulate system (5) with the parameter values as shown in Table 1.

By direct calculation, we have $R_{0}=31.4916, R_{1}=$ 30.2250, and $D(P)=0.00076142>0$ and the simulations display that the immune-present equilibrium $E^{*}$ is asymptotically stable for $\alpha=0.85, \alpha=0.95$, and $\alpha=1$ (see Figure 1). From Figure 1, we can clearly see that, compared with the case of order $\alpha=0.85$, the trajectory of model with order $\alpha=0.95$ is closer to the trajectory of the model with the integer-order 1. That is, the farther from $\alpha$ to 1 , the bigger of the trajectory difference of them. 


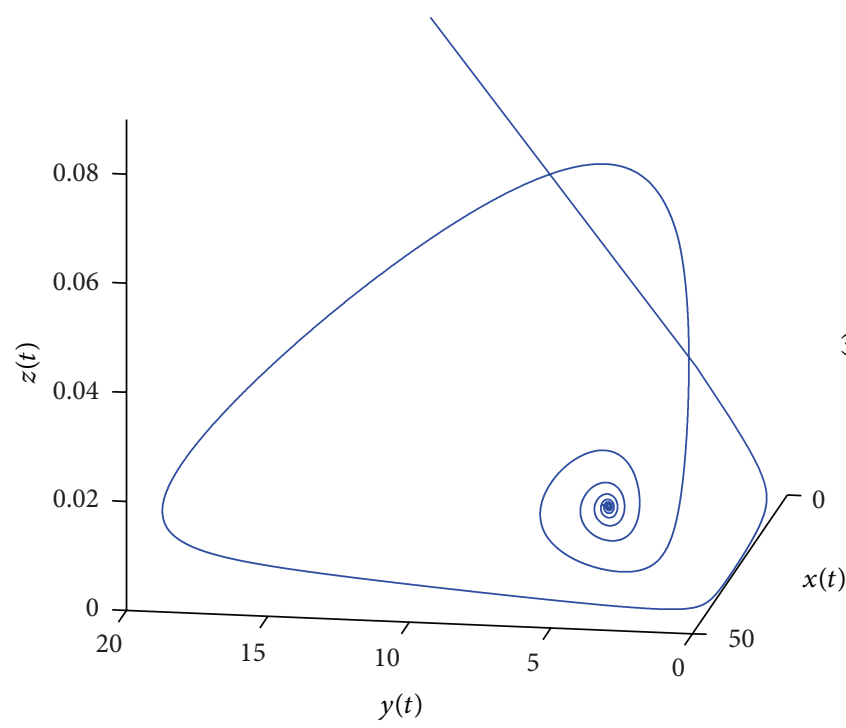

$-\alpha=0.6$

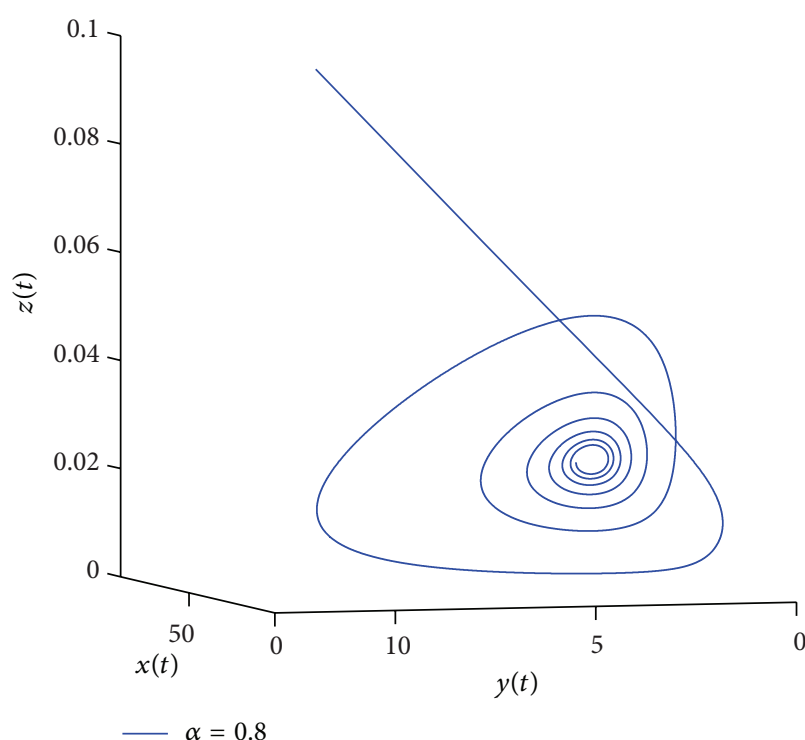

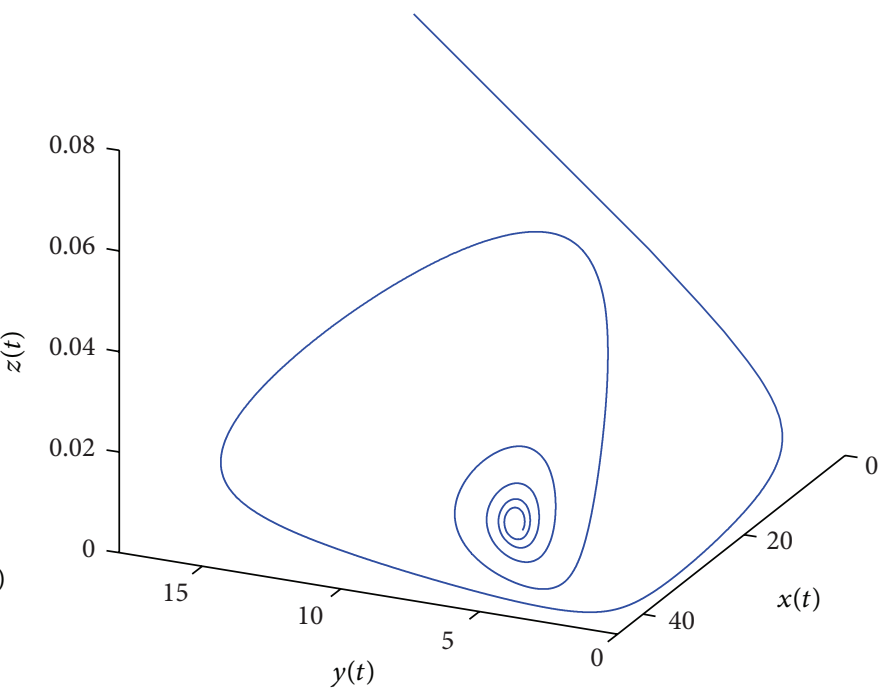

$-\alpha=0.7$

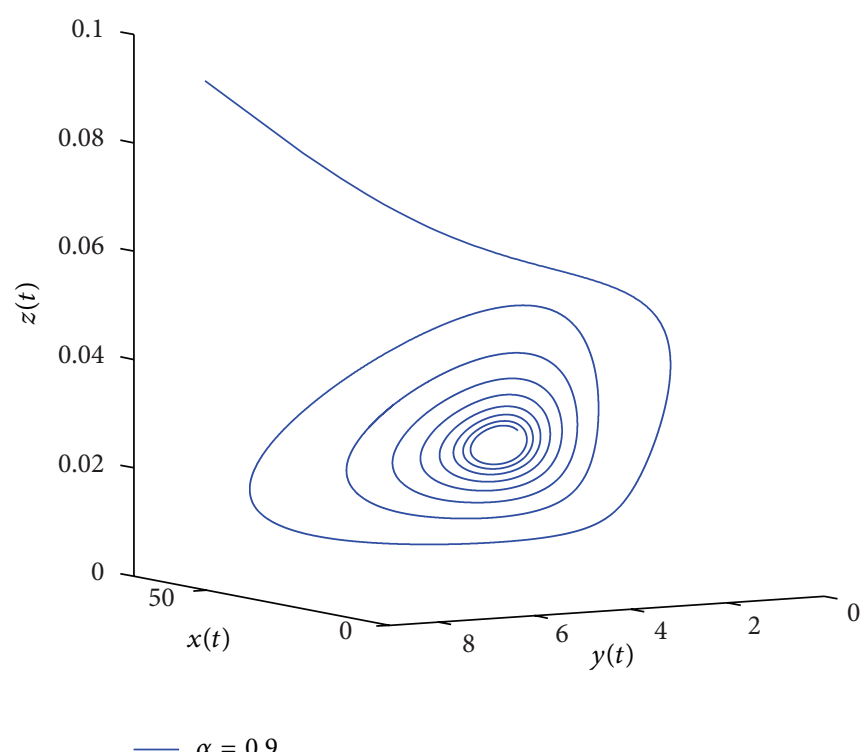

Figure 3: The three-dimensional diagrams show the approximate solutions of $x(t), y(t)$, and $z(t)$ for $\alpha=0.6, \alpha=0.7, \alpha=0.8$, and $\alpha=0.9$ in condition of $D(P)<0$.

TABle 1

\begin{tabular}{lccc}
\hline Parameter & Value & Parameter & Value \\
\hline$\lambda$ & 23.3 & $c$ & 0.0031 \\
$\beta$ & 0.5 & $d$ & 0.09 \\
$a$ & 0.02 & $p$ & 10 \\
$b$ & 0.15 & $q$ & 0.79 \\
\hline
\end{tabular}

We choose the parameter values as shown in Table 2.

Based on the parameter values in Table 2 , we have $R_{0}=$ $3.5714, R_{1}=3.4769$, and $D(P)=-0.00069<0$. With the same simulated method, it is shown that the immune-present equilibrium $E^{*}$ is asymptotically stable for $\alpha=0.56$ and $\alpha=$ 0.65 (see Figure 2).
Next, we use the method that is shown in the previous section to simulate system (5) by transforming system (5) to one order ordinary differential equation. Here we set the parameters values as shown in Table 3 with $M=10, \Delta t=$ 0.005. We have $R_{0}=9.0445, R_{1}=8.7438$, and $D(P)=$ $-10.0374<0$. The approximate solutions $x(t), y(t)$, and $z(t)$ for $\alpha=0.6, \alpha=0.7, \alpha=0.8$, and $\alpha=0.9$ are displayed in Figure 3. It shows that the immune-present equilibrium $E^{*}$ is asymptotically stable for $\alpha=0.6<2 / 3$ and there exists the limit circle for $\alpha>2 / 3$.

We also simulate the situation of system (5) by the method in the previous section when $\alpha=1$ with the parameter values of Table 3 in Figure 4. Both Figures 1 and 4 show that the immune-present equilibrium $E^{*}$ is asymptotically stable for 


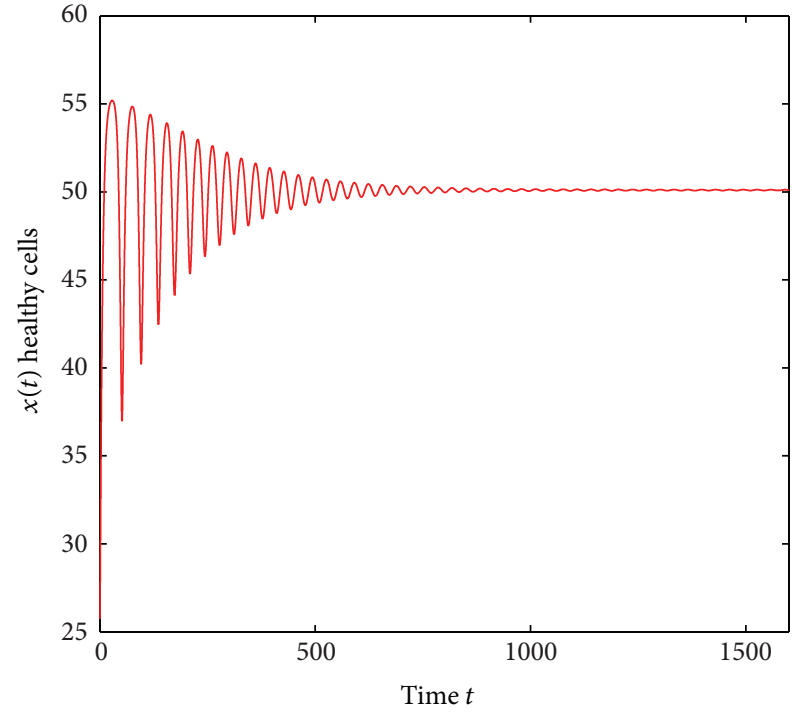

(a)

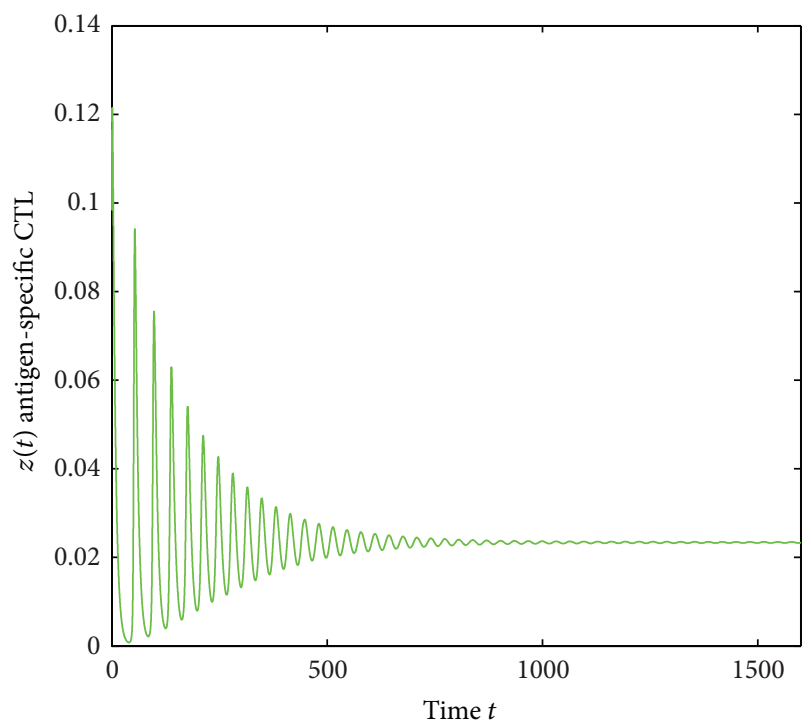

(c)

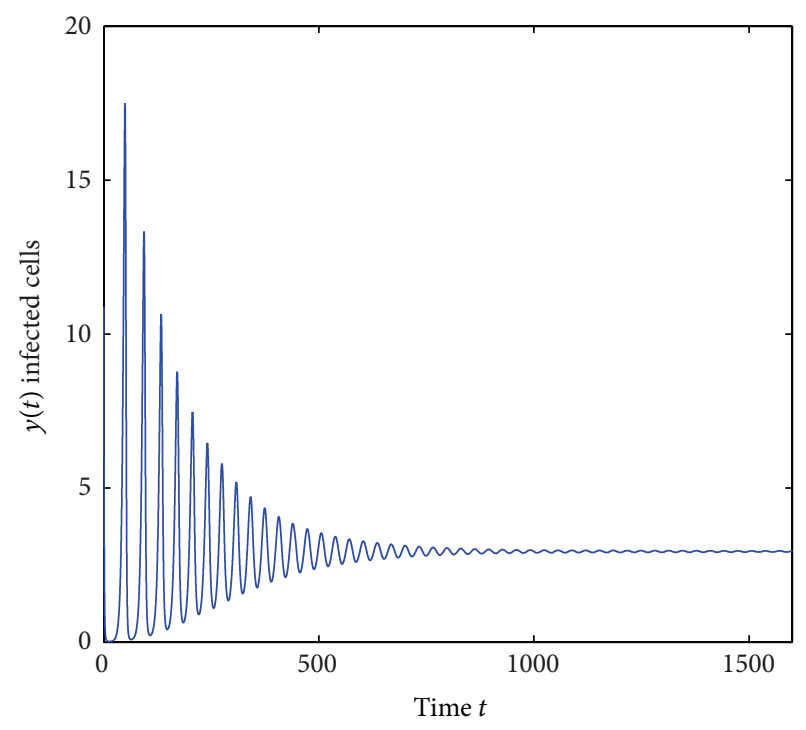

(b)

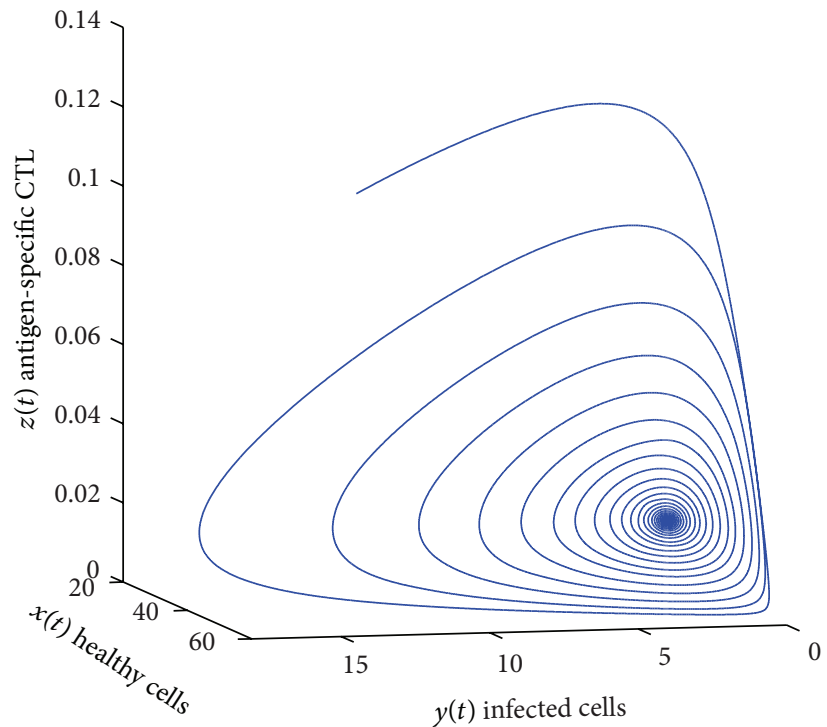

(d)

FiguRE 4: The diagrams show the approximate solutions of $x(t), y(t)$, and $z(t)$ for $\alpha=1$ in condition of $R_{1}>1$ and $D(P)<0$. The panel (a) corresponds to healthy cells, (b) to infected cells, and (c) to antigen-specific CTL. The panel (d) shows the three-dimensional trajectory of system (5) with time evolution.

TABLE 2

\begin{tabular}{lccc}
\hline Parameter & Value & Parameter & Value \\
\hline$\lambda$ & 10 & $c$ & 0.051 \\
$\beta$ & 0.01 & $d$ & 0.18 \\
$a$ & 0.1 & $p$ & 0.6 \\
$b$ & 0.15 & $q$ & 0.01 \\
\hline
\end{tabular}

$\alpha=1$ and that is nothing to do with $D(P)>0$ or $D(P)<0$. Hence the results of fractional-order system when $\alpha=1$ are consistent with the result of integer-order HIV model (4).
TABLE 3

\begin{tabular}{lccc}
\hline Parameter & Value & Parameter & Value \\
\hline$\lambda$ & 29.46 & $c$ & 0.09 \\
$\beta$ & 0.286 & $d$ & 0.9862 \\
$a$ & 0.0384 & $p$ & 0.85 \\
$b$ & 2.568 & $q$ & 0.79 \\
\hline
\end{tabular}

\section{Conclusion}

Fractional differential equations have garnered a lot of attention and appreciation due to their ability to provide an exact description of different nonlinear phenomena. 
The advantage of fractional-order systems is that they allow greater degrees of freedom in the model. Nowadays, more and more investigators begin to study the qualitative properties and numerical solutions of fractional-order virus infection models. In this paper, we introduced a fractional-order HIV infection model with nonlinear incidence and dealt with the mathematical behaviors of the model.

We showed that system (5) possesses nonnegative solutions and studied the stability behavior of the infectionfree equilibrium, the immune-absence equilibrium, and the immune-presence equilibrium. We found that the stability of the infection-free equilibrium and the immune-absence equilibrium of system (5) is the same as that of system (4). When the basic reproduction number of viruses $\left(R_{0}\right)$ is less than one, the infection-free equilibrium is stable; however, when $R_{0}$ is more than one, the infection-free equilibrium is unstable and when the immune reproduction number $\left(R_{1}\right)$ is less than one, the immune-absence equilibrium is stable; however, when $R_{1}$ is more than one, the immune-absence equilibrium is unstable. However, the results for the immunepresence equilibrium of system (5) are different to those of system (4). In system (4), the immune-presence equilibrium is stable when $R_{1}$ is more than one, while, in system (5), when $R_{1}$ is more than one, the immune-presence equilibrium is not always stable. In the condition of $R_{1}>1$, when $D(P)>0$, the immune-presence equilibrium is stable for $0<\alpha \leq 1$, while when $D(P)<0$, the immune-presence equilibrium is stable only for $0<\alpha<2 / 3$. But using the simulation, we found when $D(P)<0$, the immune-presence equilibrium is stable for $\alpha=1$. From the simulation, we also found the farther from $\alpha$ to 1 , the bigger of their trajectory difference. These results show that the integer-order model can be viewed as a special case from the more general fractional-order model. Although a large part of results is illustrated by both theory analysis and numerical simulation, the result for the immunepresence equilibrium when $D(P)<0$ and $\alpha=1$ can be just verified by the simulation in this paper.

In this paper, we introduce the fractional calculus into the HIV infection model with nonlinear incidence and, from the theory analysis and numerical simulations, it is illustrated that the integer-order HIV infection model can be viewed as a special case of fractional-order model. We hope that this work can create interest and further do research effort in this field, since the fractional modeling might provide more insight into understanding the dynamical behaviors of such systems.

\section{Conflict of Interests}

The authors declare that there is no conflict of interests regarding the publication of this paper.

\section{Acknowledgments}

This work was partially supported by the Hainan Natural Science Foundation (112006) and Natural Science Foundation of Hainan Provincial Department of Education (Hjkj2013-47). Gang Huang was supported by the Fundamental Research Funds for the Central Universities (no. CUG130415) and the National Natural Science Foundation of China (no. 11201435).

\section{References}

[1] T. M. Atanackovic and B. Stankovic, "An expansion formula for fractional derivatives and its application," Fractional Calculus \& Applied Analysis, vol. 7, no. 3, pp. 365-378, 2004.

[2] G. Bognár, "Similarity solution of boundary layer flows for nonNewtonian fluids," International Journal of Nonlinear Sciences and Numerical Simulation, vol. 10, no. 11-12, pp. 1555-1566, 2009.

[3] M. Benmalek and A. Charef, "Digital fractional order operators for R-wave detection in electrocardiogram signal," IET Signal Processing, vol. 3, no. 5, pp. 381-391, 2009.

[4] Y. Ferdi, "Some applications of fractional order calculus to design digital filters for biomedical signal processing," Journal of Mechanics in Medicine and Biology, vol. 12, no. 2, Article ID 12400088, 2012.

[5] Y. Ferdi, A. Taleb-Ahmed, and M. R. Lakehal, "Efficient generation of $1 / f^{\beta}$ noise using signal modeling techniques," IEEE Transactions on Circuits and Systems. I. Regular Papers, vol. 55, no. 6, pp. 1704-1710, 2008.

[6] R. L. Bagley and R. A. Calico, "Fractional order state equations for the control of viscoelastically damped structures," Journal of Guidance, Control, and Dynamics, vol. 14, no. 2, pp. 304-311, 1991.

[7] G. L. Jia and Y. X. Ming, "Study on the viscoelasticity of cancellous bone based on higher-order fractional models," in Proceedings of the 2nd International Conference on Bioinformatics and Biomedical Engineering (ICBBE '08), pp. 1733-1736, 2006.

[8] J. H. He, "Some applications of nonlinear fractional differential equations and their approximations," Bulletin of Science Technology \& Society, vol. 15, pp. 86-90, 1999.

[9] B. Mandelbrot, "Some noises with $1 / f$ spectrum, a bridge between direct current and white noise," IEEE Transactions on Information Theory, vol. 13, no. 2, pp. 289-298, 1967.

[10] R. L. Magin, "Fractional calculus in bioengineering," Critical Reviews in Biomedical Engineering, vol. 32, pp. 1-377, 2004.

[11] Y. A. Rossikhin and M. V. Shitikova, "Applications of fractional calculus to dynamic problems of linear and nonlinear hereditary mechanics of solids," Applied Mechanics Reviews, vol. 50, no. 1, pp. 15-67, 1997.

[12] F. Mainardi, "Fractional calculus: some basic problems in continuum and statistical mechanics," in Fractals and Fractional Calculus in Continuum Mechanics, vol. 378, pp. 291-348, Springer, Berlin, Germany, 1997.

[13] R. T. Baillie, "Long memory processes and fractional integration in econometrics," Journal of Econometrics, vol. 73, no. 1, pp. 559, 1996.

[14] K. S. Cole, "Electric conductance of biological systems," in Proceedings of the Cold Spring Harbor Symposia on Quantitative Biology, pp. 107-116, Cold Spring Harbor, NY, USA, 1993.

[15] V. D. Djordjević, J. Jarić, B. Fabry, J. J. Fredberg, and D. Stamenović, "Fractional derivatives embody essential features of cell rheological behavior," Annals of Biomedical Engineering, vol. 31, no. 6, pp. 692-699, 2003.

[16] R. V. Culshaw and S. Ruan, "A delay-differential equation model of HIV infection of $\mathrm{CD}^{+}$T-cells," Mathematical Biosciences, vol. 165, no. 1, pp. 27-39, 2000.

[17] G. Huang, H. Yokoi, Y. Takeuchi, T. Kajiwara, and T. Sasaki, "Impact of intracellular delay, immune activation delay and nonlinear incidence on viral dynamics," Japan Journal of Industrial and Applied Mathematics, vol. 28, no. 3, pp. 383-411, 2011. 
[18] G. Huang, Y. Takeuchi, and A. Korobeinikov, "HIV evolution and progression of the infection to AIDS," Journal of Theoretical Biology, vol. 307, pp. 149-159, 2012.

[19] A. S. Perelson, D. E. Kirschner, and R. de Boer, "Dynamics of HIV infection of CD4 ${ }^{+}$T cells," Mathematical Biosciences, vol. 114, no. 1, pp. 81-125, 1993.

[20] A. S. Perelson and P. W. Nelson, "Mathematical analysis of HIV1 dynamics in vivo," SIAM Review, vol. 41, no. 1, pp. 3-44, 1999.

[21] A. A. M. Arafa, S. Z. Rida, and M. Khalil, "Fractional modeling dynamics of HIV and CD4 $4^{+}$T-cells during primary infection," Nonlinear Biomedical Physics, vol. 6, article 1, 2012.

[22] Y. Ding and H. Ye, "A fractional-order differential equation model of HIV infection of $\mathrm{CD}^{+}{ }^{+}$T-cells," Mathematical and Computer Modelling, vol. 50, no. 3-4, pp. 386-392, 2009.

[23] A. Korobeinikov, "Global properties of infectious disease models with nonlinear incidence," Bulletin of Mathematical Biology, vol. 69, no. 6, pp. 1871-1886, 2007.

[24] A. Korobeinikov, "Global asymptotic properties of virus dynamics models with dose dependent parasite reproduction and virulence, and nonlinear incidence rate," Mathematical Medicine and Biology, vol. 26, pp. 225-239, 2009.

[25] A. Korobeinikov, "Stability of ecosystem: global properties of a general predator-prey model," Mathematical Medicine and Biology, vol. 26, no. 4, pp. 309-321, 2009.

[26] I. Petras, Fractional-Order Nonlinear Systems: Modeling, Analysis and Simulation, Springer, New York, NY, USA, 2011.

[27] E. Ahmed and A. S. Elgazzar, "On fractional order differential equations model for nonlocal epidemics," Physica A: Statistical Mechanics and Its Applications, vol. 379, no. 2, pp. 607-614, 2007.

[28] Z. M. Odibat and N. T. Shawagfeh, "Generalized Taylor's formula," Applied Mathematics and Computation, vol. 186, no. 1, pp. 286-293, 2007.

[29] W. Lin, "Global existence theory and chaos control of fractional differential equations," Journal of Mathematical Analysis and Applications, vol. 332, no. 1, pp. 709-726, 2007.

[30] T. M. Atanackovic and B. Stankovic, "On a numerical scheme for solving differential equations of fractional order," Mechanics Research Communications, vol. 35, no. 7, pp. 429-438, 2008. 


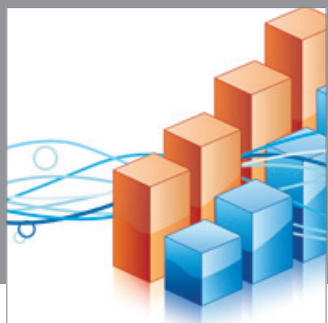

Advances in

Operations Research

mansans

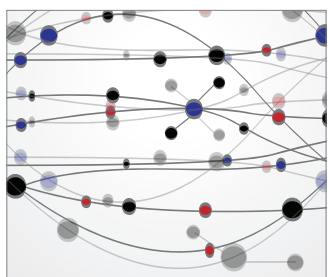

The Scientific World Journal
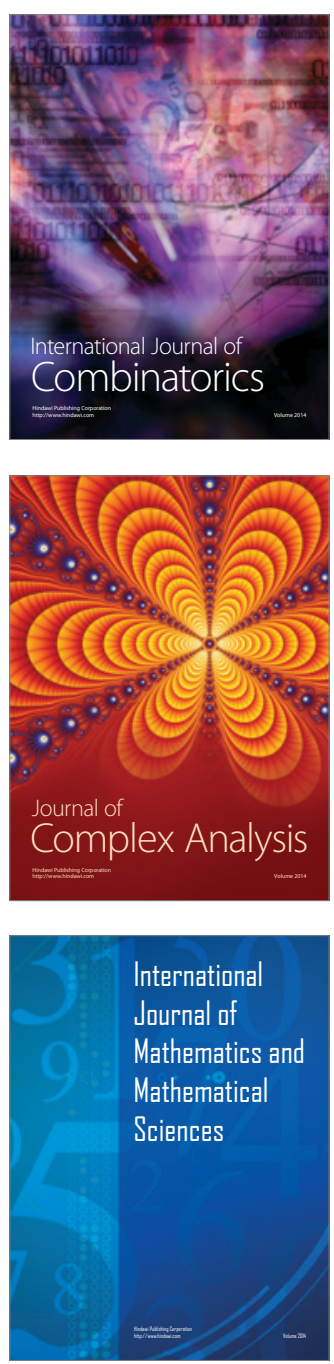
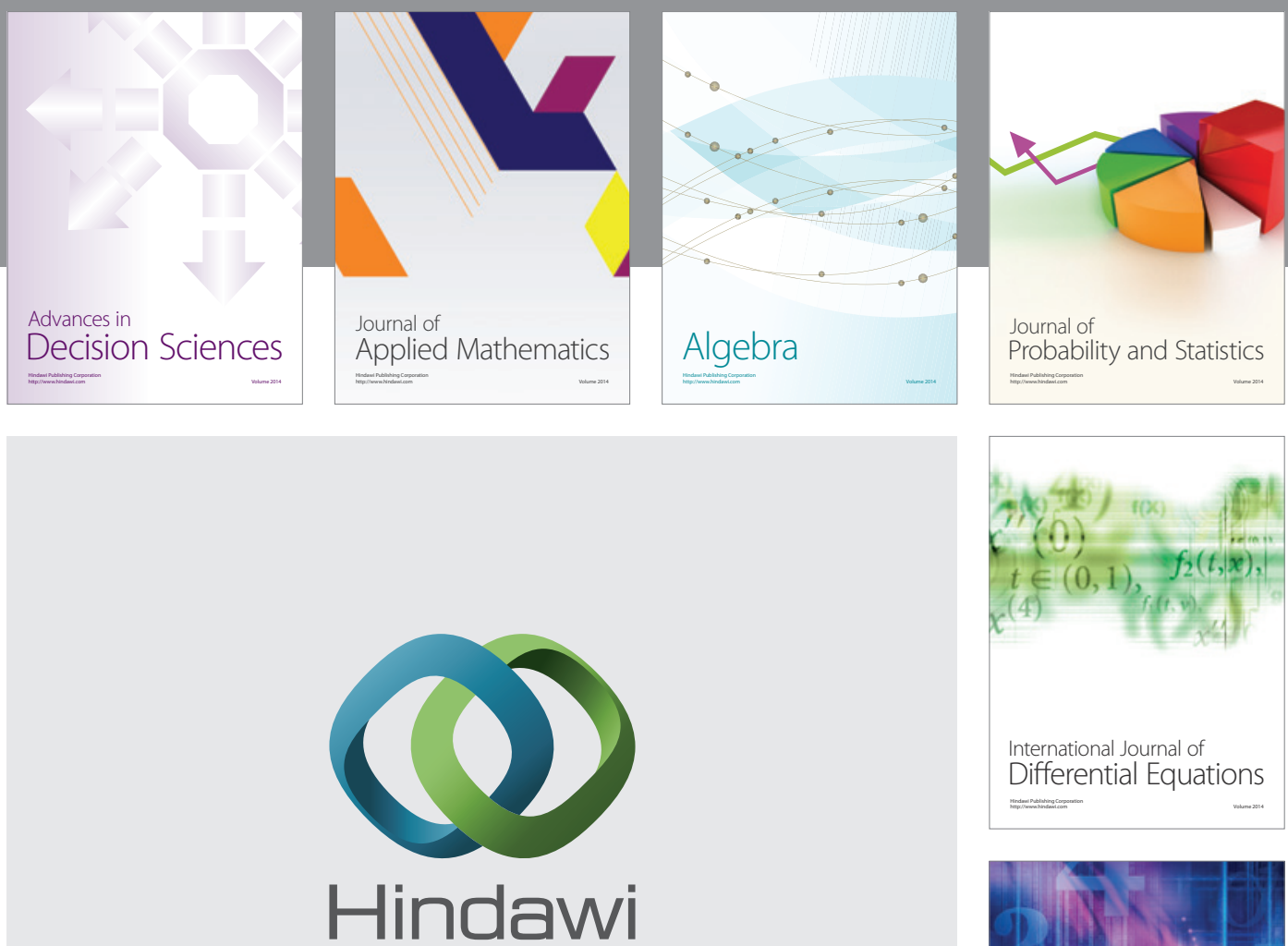

Submit your manuscripts at http://www.hindawi.com
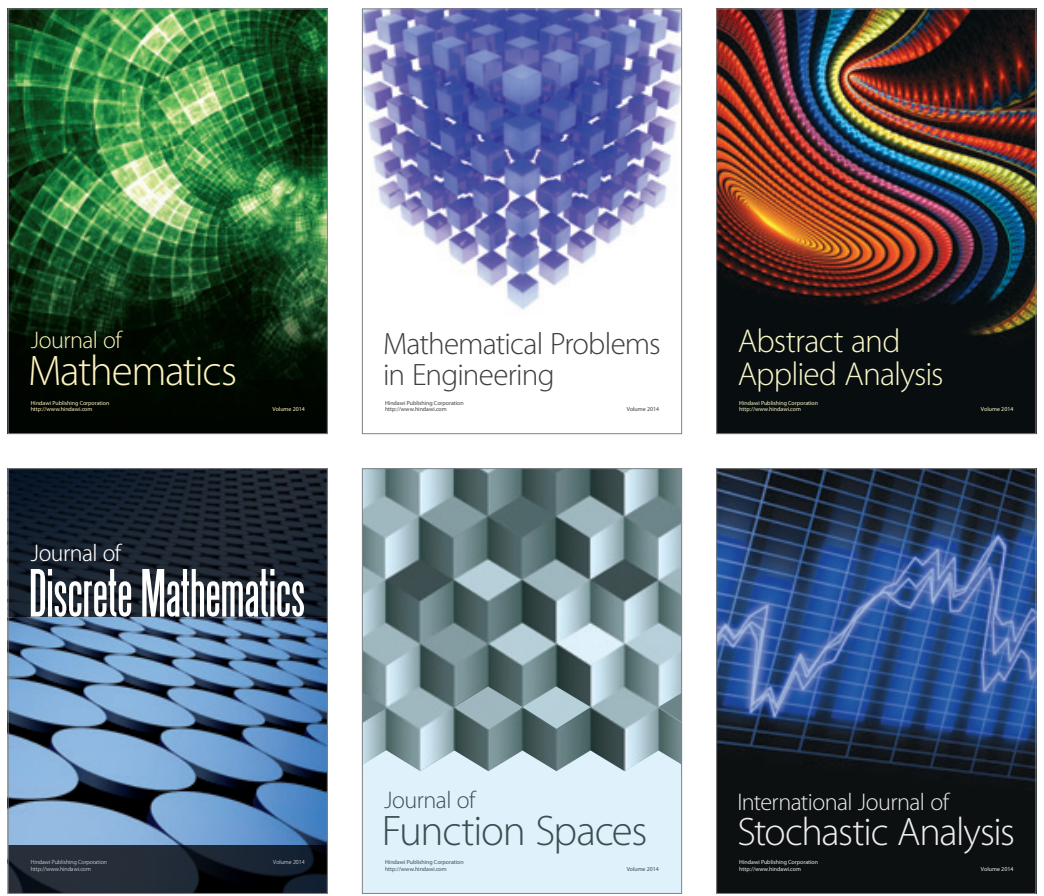

Journal of

Function Spaces

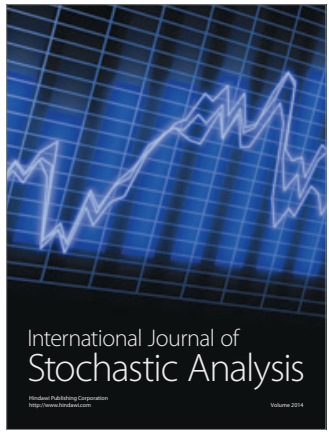

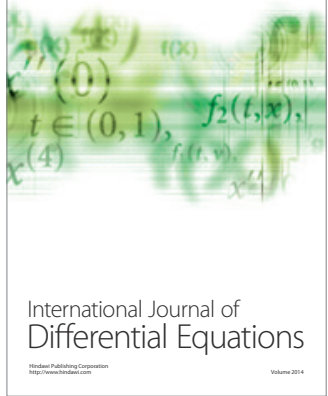
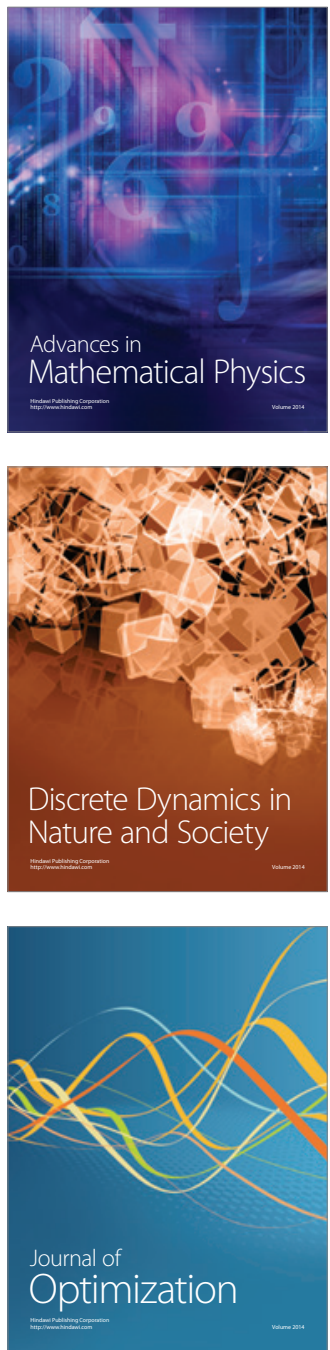